\title{
Active Contours with Term of Smoothing: Application in Medical Imaging
}

\author{
RIAD DIB \\ Akli Mohand Oulhadj University, Bouira, ALGERIA
}

\begin{abstract}
The segmentation of images is to group the different pixels of the image into classes, each class grouping similar pixels in the sense of a given criteria. The segmentation of images is, in general, very difficult to achieve because the natural images are various, complex and the way of perceiving them varies according to the individuals. Because of this difficulty, several approaches have been proposed. The approach addressed in this article is the technique of active contours. We focus on implicit active contours; we propose an improvement for the model of Chan\&Vese. The application of our method on medical images shown they can be a tool for the image segmentation, and thus provide an aid to diagnosis for the practitioner.
\end{abstract}

Key Words: Image segmentation; implicit active contours; level set; medical imaging

Received: February 10, 2020. Revised: August 1, 2020. Accepted: August 13, 2020. Published: August 27, 2020.

\section{Introduction}

The method of active contours is one of the effective methods for the segmentation of images [1-7]. Active contours can be divided into two approaches: approaches "contours" [1,3-6] and approaches "regions" [8-10]. These two approaches have advantages and disadvantages; the choice of the approach in an application depends on various types and characteristic of the images.

The approach "contours" uses the gradient of the image to prevent the evolution of contour on the borders of the image to be segmented. This approach requires a stopping function and a balloon force to inspect the movement of active contour. The stop function is used to stop contour on the desired object. The force of the balloon is involved to inflate or deflate contour (curve of evolution). The choice of this force raises sometimes difficulties: if we selected the force very small, curve does not manage to locate the object of interest and in the opposite case, the curve can exceed the borders of weak contrast of the object of interest.

The approach "regions" has advantages compared to the approach "contours". First, the approach "regions" does not use the gradient of the image but it uses the regional statistics. Second, this approach is not sensitive to initialization (does not depend on the initial position of the curve).

The most popular model in this approach is that of Chan\&Vese [8]. This model offers satisfactory results for the segmentation of an image which presents two regions of almost uniform intensities, the model assumes that the image to be segmented is made up of the homogeneous regions and in the case or the image is made up of the inhomogeneity regions, the model of Chan\&Vese becomes inadequate for the segmentation of such images.

In this article we propose a new improved version for the model of Chan\&Vese for the segmentation of the images with various intensity. Our model uses a Gaussian function to define the 
local energy of refinement in the variational formulation of the model. Local intensity information can be inserted in the model suggested by Li [5], the reinitialization of contour is not necessary for our method.

\section{Implicit active contours}

This type of active contours at summer implemented to cure the problems arising from the method of explicit active contours (Snakes) [3,11]. The method of implicit active contours calls upon the theory of level sets.

The principle of the level sets is inspired by the theory of the propagation of the curves undergoing a normal force. The first work relative to this method is those of Dervieux and Thomasset in connection with the interfaces between fluids of nothomogeneous density moving $[12,13]$. Thereafter, Osher and Sethian modelled the propagation of the interfaces (or faces) between fluids and/or different solids of nature under the action of dependent force on their curvatures $[14,15]$.

\subsection{Approaches Mumford-Shah}

In $2 \mathrm{D}$, let $\Omega$ be the space of the image, and $\mathrm{C}$ is a curve defining a region of the image w of $\Omega(\mathrm{w} \in \Omega$ and $\mathrm{C}=\partial \mathrm{w}$ ). We denote by $\mathrm{I}: \Omega \rightarrow \mathrm{R}$ the given image and $L(C)$ the length of curve $C$. The energy function of Mumford and Shah [16] is then given by:

$$
\begin{gathered}
E^{M S}(u, C)= \\
\lambda \int_{\Omega}(\mathrm{I}-u)^{2} d x d y \int_{\Omega \backslash C}|\nabla u|^{2} d x d y+\mu L(C)
\end{gathered}
$$

where $\mu, \lambda>0$ is fixed parameters used to measure the weight of the various terms of energy.
It is seen that the parameter $\mu$ influences over the length of the border. Thus one will want details, more it will be necessary to decrease this parameter. This returns the minimization of the functional calculus of energy (1) is difficult.

\subsection{Chan\&Vese models}

Chan and Vese have proposed an active contour approach for the Mumford-Shah problem in a particular case where the image $u$ is defined in (1) is an almost constant function $[17,18]$.

The basic idea of this model is simple, since the assumption is made that image $I$ is made up of two regions of almost uniform intensities $\mathrm{u}_{0}^{\mathrm{i}}$ and $\mathrm{u}_{0}^{\mathrm{e}}$.

Starting from this hypothesis, we can say that I = $\mathrm{u}_{0}^{\mathrm{i}}$ in the region $\omega$ and $\mathrm{I}=\mathrm{u}_{0}^{\mathrm{e}}$ in the region outside $\omega$. We now consider the following refinement energy term $[8,19-20]$ :

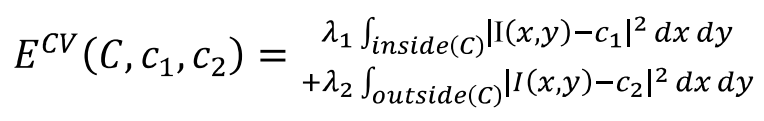

where inside $(C)$ and outside $(C)$ represent the region inside and outside of the contour $\mathrm{C}$, respectively, and $\mathrm{c} 1$ and $\mathrm{c} 2$ are two constants that approximate the image intensity in inside(C) and outside(C).

Into this model, Chan and Vese [8,19-20] introduced two terms of regularization to minimize the length of the curve and the internal surface of the curve $\mathrm{C}$, which introduces the energy functional E:

$$
\begin{aligned}
& E^{C V}\left(C, c_{1}, c_{2}\right)= \\
& \begin{array}{c}
\mu L(C)+\quad+\lambda_{1} \int_{\text {inside }(C)}\left|\mathrm{I}(x, y)-c_{1}\right|^{2} d x d y \\
v A(\text { inside }(C)) \\
+\lambda_{2} \int_{\text {outside }(C)}\left|\mathrm{I}(x, y)-c_{2}\right|^{2} d x d y
\end{array}
\end{aligned}
$$


with, $\mu \geq 0, v \geq 0$ and $\lambda_{1}, \lambda_{2}$ parameters balancing the various terms of the functional energy.

The goal of this model is to minimize energy E. This problem of minimization can be formulated and solved by using the method of the level sets.

The minimization of the equation (3) by using the formulation of level sets, amounts solving the partial derivative equation following [8]:

$$
\begin{aligned}
& \frac{\partial \Phi}{\partial t}=\delta_{\varepsilon}(\Phi)\left[\mu \operatorname{div}\left(\frac{\nabla \Phi}{|\nabla \Phi|}\right)-v-\lambda_{1}\left(I-c_{1}\right)^{2}+\right. \\
& \lambda 2 \digamma c 22
\end{aligned}
$$

The application of this model on images tests shown that is not sensitive to the noise and it automatically manages the changes of topology in the images. If the two regions with interior and external of the curve $\mathrm{C}$ are not homogeneous (with various intensity), this model poses problems of segmentation for some parts of the images.

On the image of the blood vessel, the intensity of the background (bottom) of the image gradually decreases from the top to the base. In addition, a part of the bottom (upper left corner) is of higher intensity than part of the blood vessel (bottom left of the branch).

Figure 1 indicates the results of segmentation per threshold and by using the model of Chan\&Vese respectively. It is noticed clearly that the blood vessel is badly segmented, as long as the image does not have a uniformity of intensity between the image of the vessel and the bottom.
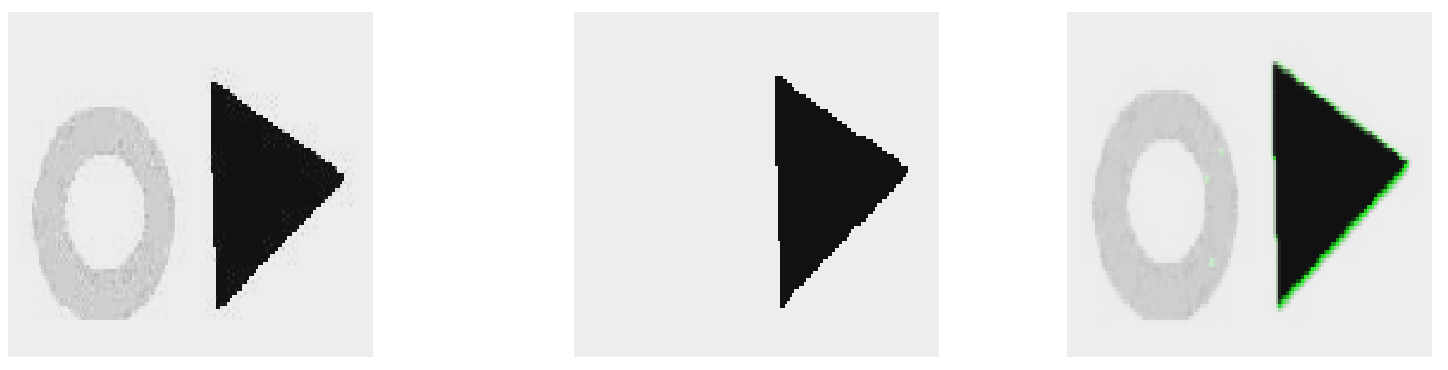

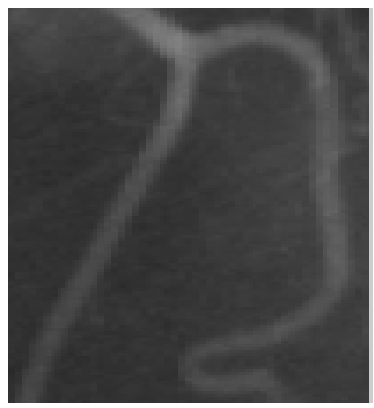

(a)

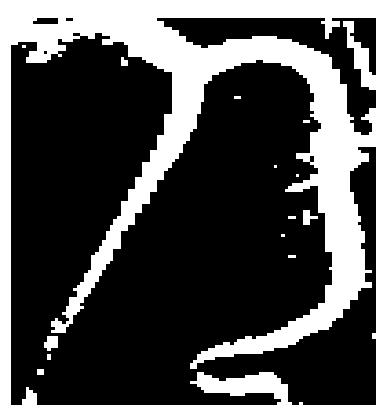

(b)

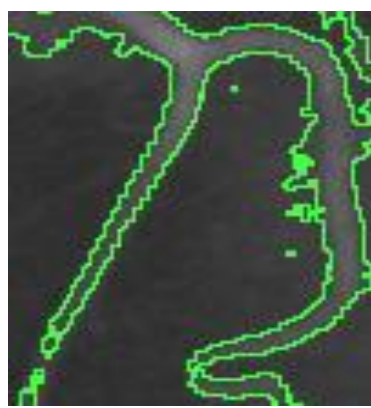

(c)

Fig. 1. Results of segmentation: a. original images, b. thresholding results, c. model of Chan\&Vese.

Similarly, Chan\&Vese [21] proposed another method, consists in using several functions of level sets. This style does not manage to solve the problem of inhomogeneity of the images. Moreover, this last model thus requires a reinitialization for the function of level sets $\Phi$. this model uses several parameters which will be difficult to select by a user not

expert. 


\section{Chan \& Vese model with binary local refinement term}

\subsection{Local refinement energy}

Consider the given imageI: $\Psi \rightarrow \mathbb{R}^{d}$, where $\Psi \subset \mathbb{R}^{n}$ the domain of the image, d is is the dimension of the vector $I(x)$. In our case $d=1$ for a grayscale image. Let $\mathrm{C}$ be the contour in the image domain $\Psi$.

We define for each point $x \in \Psi$ the following energy term[21]:

$$
\begin{gathered}
E^{R L B}\left(C, c_{1}(x), c_{2}(x)\right)= \\
\lambda_{1} \int_{\operatorname{int}(C)} K(x-y)\left|\mathrm{I}(y)-c_{1}(x)\right|^{2} d x d y \\
+\lambda_{2} \int_{\operatorname{ext}(C)} K(x-y)\left|\mathrm{I}(y)-c_{2}(x)\right|^{2} d x d y
\end{gathered}
$$

Where, $\lambda_{1}$ and $\lambda_{2}$ are positive parameters, $\mathrm{K}$ is the kernel function, where $\mathrm{K}(\mathrm{x})$ decreases and approaches zero as long as $\mathrm{x}$ increases, $\mathrm{c} 1(\mathrm{x})$ and $\mathrm{c} 2$ (x) are the two terms that correspond to image intensities Of the point $\mathrm{x}$.

In our work, we choose the function of the Gaussian kernel:

$$
K_{\sigma}(\alpha)=\frac{1}{\sqrt{2 \pi} \sigma} \mathrm{e}^{-\frac{\alpha^{2} / 2}{2 \sigma^{2}}}
$$

With $\sigma$ is the standard deviation. The right choice of this parameter is very important for a good location of the objects of interest.

The introduction of the two variant functions $\mathrm{c} 1$ and c2 makes our method essentially different from that of Chan \& Vese [8].

As a consequence, the image intensities at points $y$ near $x$ have a great influence on the values of the two functions $\mathrm{c} 1$ and $\mathrm{c} 2$ which minimizes the energy function $E^{\mathrm{RLB}}\left(\mathrm{C}, \mathrm{c}_{1}(\mathrm{x}), \mathrm{c}_{2}(\mathrm{x})\right)$, while away from the point $\mathrm{x}$, the image intensities do not influence $\mathrm{cl}$ and $\mathrm{c} 2$.

Thus, for any point $\mathrm{x}$, the local refinement energy $E^{R L B}\left(C, c_{1}(x), c_{2}(x)\right)$ can be minimized when the contour $\mathrm{C}$ locates the boundary of the object to be detected for an optimal choice of $\mathrm{c} 1$ and c2.

Our objective is to minimize $\mathrm{E}^{\mathrm{RLB}}\left(\mathrm{C}, \mathrm{c}_{1}(\mathrm{x}), \mathrm{c}_{2}(\mathrm{x})\right)$ for all points $\mathrm{x}$ in the image domain $\Omega$. For this purpose the energy function defined in (5) can be converted into an equivalent level set formulation, of which a new model of the active contours will be obtained.

\subsection{Formulation of the model with the levels sets}

In the level sets method, the contour is represented by the zero level of a signed distance function $\Phi: \Psi \rightarrow \mathrm{R}$, such that $[23,24]$ :

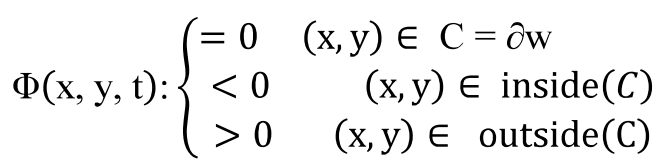

Using the representation of the level sets, the energy function $\mathrm{E}^{\mathrm{RLB}}\left(\mathrm{C}, \mathrm{c}_{1}(\mathrm{x}), \mathrm{c}_{2}(\mathrm{x})\right)$ can be written as follows:

$$
\begin{gathered}
E^{R L B}\left(\Phi, c_{1}(x), c_{2}(x)\right)= \\
\lambda_{1} \int_{\Omega} K_{\sigma}(x-y)\left|\mathrm{I}(y)-c_{1}(x)\right|^{2} \mathrm{H}(\Phi(y)) d y d x+ \\
\lambda_{2} \int_{\Omega} K_{\sigma}(x-y)\left|\mathrm{I}(y)-c_{2}(x)\right|^{2}(1-\mathrm{H}(\Phi(y))) d y d x
\end{gathered}
$$

We denote by $\mathrm{H}$ the Heaviside function $[8,17]$. With an aim of ensuring a stable evolution for the curve of evolution $\Phi$, one adds a term of regularization of distance proposed by Li [5]. 
This term is given as follows:

$$
\mathrm{P}(\Phi)=\int_{\Omega} \frac{1}{2}(|\nabla \Phi(x)|-1)^{2} d x d y
$$

To regularize the zero level contour of $\Phi$, we also need the length of the zero level of $\Phi$, which is given by

$$
L(\Phi)=\int_{\Omega} \delta(\Phi(x))|\nabla(\Phi(x))| d x d y
$$

Now, we define the entire energy functional

$$
f\left(\Phi, \mathrm{c}_{1}, \mathrm{c}_{2}\right)=E^{R L B}\left(\Phi, c_{1}, c_{2}\right)+\mu \mathrm{P}(\Phi)+\rho L(\Phi)
$$

where $\mu$ and $\rho$ are positive constants.

In order to minimize the energy function (11), we first have to regularize the Heaviside $\mathrm{H}$ function in equation (8) and the Dirac function $\delta$ in equation (10), Chan and Vese [20] propose:

$$
\begin{aligned}
& H_{\varepsilon}(\Phi)=\frac{1}{2}+\frac{1}{\Pi} \arctan \left(\frac{\Phi}{\varepsilon}\right) \\
& \delta_{\varepsilon}(\Phi)=\frac{1}{\Pi} \frac{\varepsilon}{\varepsilon^{2}+\Phi^{2}}
\end{aligned}
$$

By replacing $\mathrm{H}$ and $\delta$ in (8) and (10) with $\mathrm{H} \varepsilon$ and $\delta_{\varepsilon}$, the functional energy $E^{R L B}$ and $\mathrm{L}$ are regularized as e $E_{\varepsilon}^{R L B}$ and $L \varepsilon$. As in [2,12], we choose $\varepsilon=1.0$ for good approximation of $\mathrm{H}$ and $\delta$ by $\mathrm{H} \varepsilon$ and $\delta_{\varepsilon}$. Thus, the functional energy $f\left(\Phi, \mathrm{c}_{1}, \mathrm{c}_{2}\right)$ in (11) is calculated by

$$
f_{\varepsilon}\left(\Phi, c_{1}, c_{2}\right)=E_{\varepsilon}^{R L B}\left(\Phi, c_{1}, c_{2}\right)+\mu \mathrm{P}(\Phi)+
$$$$
\rho L_{\varepsilon}(\Phi)
$$

\subsection{Gradient descent flow}

The standard gradient descent method (or Steepest descent method) is used to minimize the energy functionality defined in (14). The flow derivation of

\subsection{The most of our method}

Contrary to the Chan \& Vese model [17], in our method, the regularization of the two refinement functions $\mathrm{c} 1$ and $\mathrm{c} 2$ is not necessary. In fact, $\mathrm{c} 1$ and c2 are smoothing functions due to the presence of the Gaussian convolution in both formulations (13) and (14). Another advantage is that our method does the gradient is identical to that of the Chan and Vese model [21]. For this purpose we must calculate the variations of the two functions $\mathrm{c} 1(\mathrm{x})$ and $\mathrm{c} 2(\mathrm{x})$ which minimize $f_{\varepsilon}\left(\Phi, \mathrm{c}_{1}, \mathrm{c}_{2}\right)$ which introduces $[20,23,25]$ :

$$
\begin{aligned}
c_{1}(\mathrm{x}) & =\frac{K_{\sigma}(x) *\left[H_{\varepsilon}(\Phi(\mathrm{x})) \mathrm{I}(x)\right]}{K_{\sigma}(x) * H_{\varepsilon}(\Phi(\mathrm{x}))} \\
c_{2}(x) & =\frac{K_{\sigma}(x) *\left[\left(1-H_{\varepsilon}(\Phi(x))\right) I(x)\right]}{K_{\sigma}(x) *\left(1-H_{\varepsilon}(\Phi(x))\right)}
\end{aligned}
$$

According to the equations (15) and (16), we note that the denominators are always positive.

We can then deduce the partial differential equation (PDE) of the energy equation (14) using the gradient descent flow:

$$
\begin{aligned}
& \frac{\partial \Phi}{\partial t}-\delta_{\epsilon}(\Phi)\left(\lambda_{1} f_{1}-\lambda_{2} f_{2}\right)++\rho \delta_{\epsilon}(\Phi) \operatorname{div}\left(\frac{\nabla \Phi}{|\nabla \Phi|}\right)+ \\
& \mu^{2}\left(\nabla^{2} \Phi-\operatorname{div}\left(\frac{\nabla \Phi}{|\nabla \Phi|}\right)\right)
\end{aligned}
$$

where $\delta_{\epsilon}$ are the smooth Dirac function given by (13), and $\mathrm{f} 1$ and $\mathrm{f} 2$ are the functions as below $f 1(x)=\int_{\Omega} K_{\sigma}(x-y)\left|\mathrm{I}(y)-c_{1}(x)\right|^{2} d x d y$

and

$$
f_{2}(x)=\int_{\Omega} K_{\sigma}(x-y)\left|\mathrm{I}(y)-c_{2}(x)\right|^{2} d x d y
$$

where $c_{1}$ and $c_{2}$ are given by (15) and (16), respectively.

Therefore, equation (17) implements our model of implicit active contour by improving the one implemented by Chan \& Vese proposed in the article [17].

not require a reset of $\Phi$ due to the regularized distance term defined in equation (7). Practically, one can simply initialize the curve $\Phi$ as a binary function, which takes the constant value $\mathrm{c} 0$ in the region $\mathrm{w}$ and $-\mathrm{c} 0$ outside of $\mathrm{w}$, where $\mathrm{w}$ can be given arbitrarily as a subset in the image domain $\Omega$. 


\section{Experimental results}

The partial derivatives $\partial \Phi / \partial \mathrm{x}$ and $\partial \Phi / \partial \mathrm{y}$ in (17) are discretized using the finite difference scheme in [6].

Our method is used on synthetic and real images of different topologies. We use the same parameters of $\rho=0.001 \times 255^{2}$, time $\tau=0.1, \mu=1.0$, and $\mu=1.0, \lambda_{1}=\lambda_{2}=1.0, \sigma=3.0$ for the image set in this work, unless the retina image show in figure 5, for which we set $\rho=10^{-10} \times 255^{2}$.

Figure 2 shows the results of segmentation by our model on synthetic and real images (blood-

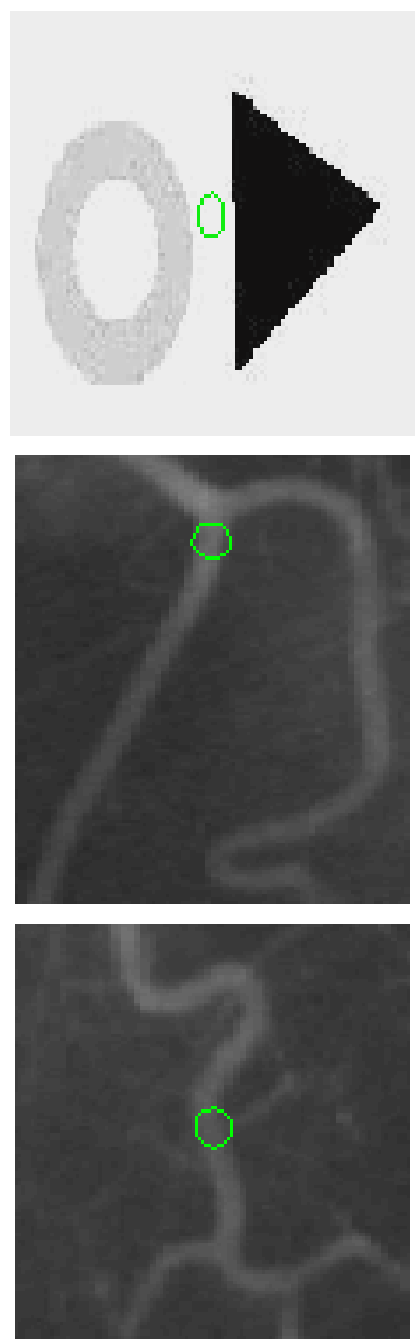

(a) vessels). The initial curves and the final curves are represented on the left and on the right respectively. In these images, it not uniformity of intensities appears both the background and the foreground.

It should be noted that the synthetic image on the first line and the image of the blood-vessel on the second line are the same images represented on figure 1 , this last watch which the model of Chan\&Vese fails to locate correctly the various branches for the real image and the object of weak contrast for the synthetic image.
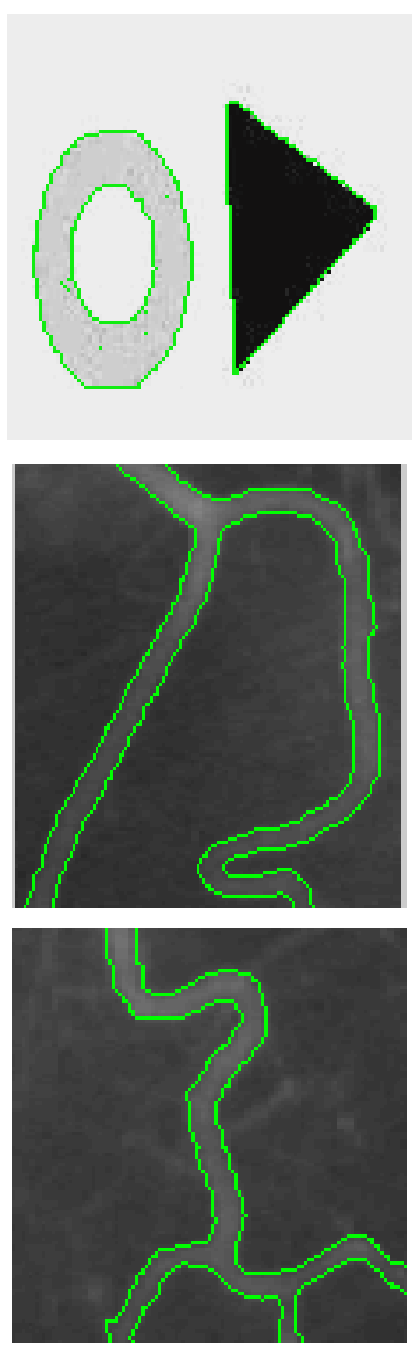

(b)

Fig. 2. Results of segmentation: (a). original images, (b). results by our method. 
On the images of the blood-vessels, some parts of the vessels are weak contrasts. Like famous figure 1, our method carries out results of satisfactory segmentation for the three images. The objects of weak contrasts and the inhomogeneity regions of the blood vessels are well detected.

Figure 3 indicate the result of our model on a noisy synthetic image with three objects and with intensity that is not homogeneous. For this image, the initial curve is sited beside an object and background of the image as on the left column of figure 3.The figure shows that final contour recovers perfectly the borders of the three objects. We note

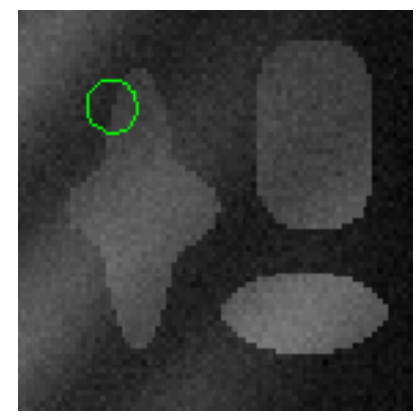

(a) that our method is effective for the segmentation of several objects with homogeneous regions or not.

Another typical example of inhomogeneity images is shown on figure 4. Filing represents the MRI of the retina; the image is of topology very complex. The retinal vessels are different sizes and from contrast varied (some vessels have contrast very close to that of the background).

The application of our model on the image of the retina shows the solidity of the method for the segmentation of the complex images. The various retinal vessels (large or small) are detected goods, in the same way for the vessel of weak contrasts.

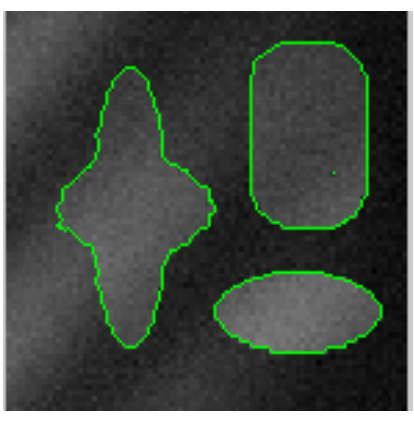

(b)

Fig. 3. Segmentation result of our model on a noisy synthetic image: (a). original image, (b), results by our method

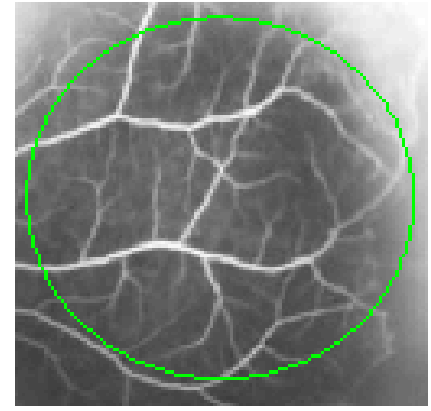

(a)

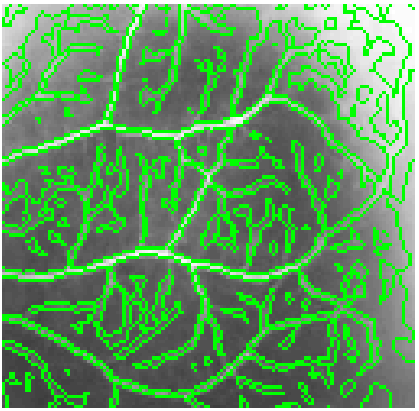

(b)

Fig. 4. Segmentation result of our model on MRI of the retina: (a). original image, (b). results by our method 


\section{Comparison with the model of Chan\&Vese}

Figure 5 shows the results of our method (third column) and that of Chan\&Vese (second column) using the same initial contour (the first column). The time of convergence (CPU times) of two methods is shown on table 1 . The results are obtained with a process Pentium $2 \mathrm{GHz}, 2 \mathrm{~GB}$ of RAM, with Matlab 6.5.

Table 1 CPU time (as a second) for two methods of segmentation for the images of figure 5 in the order

\begin{tabular}{l|ccc}
\hline Methods & Img1 & Img2 & Img3 \\
\hline Model of Chan\&Vese & 108.92 & 114.89 & 665.64 \\
Our model & 3.547 & 3.89 & 120,32
\end{tabular}

It is noticed clearly that our model is faster of that of Chan\&Vese, according to table 1; our method is quick than the Chan\&Vese methods. This

Also our model is higher in terms of precision, due to its capacity to use the local information of image. This is shown on the image of the retina (figure5).

Our method manages to extract the various retinal vessels from contrasts and sizes different, which is not the case for the model of Chan\&Vese; this last does not manage to extract the vessels from weak contrasts and small sizes. Moreover, some undesirable contours are generated by the model of Chan\&Vese. Our method effectively manages to cure this problem, which is shown on the images of the blood vessel. demonstration shows the benefit of our method in form of effectiveness of time of convergence. 

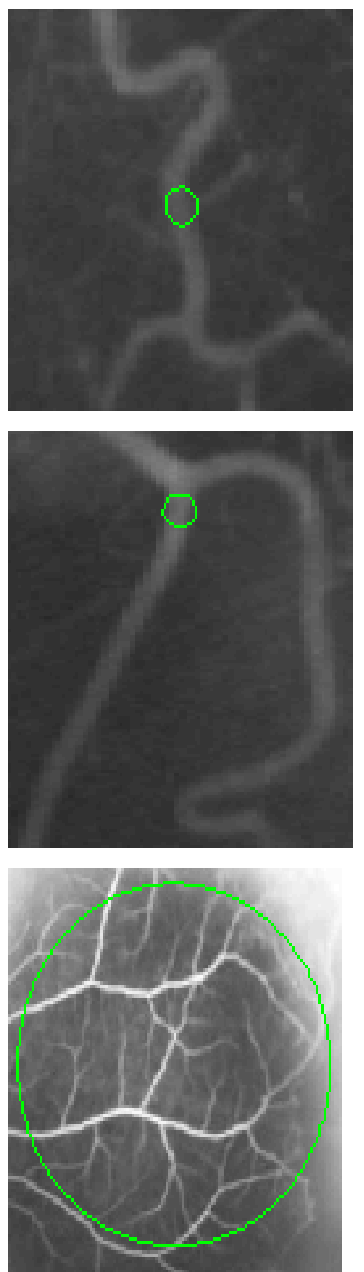

(a)
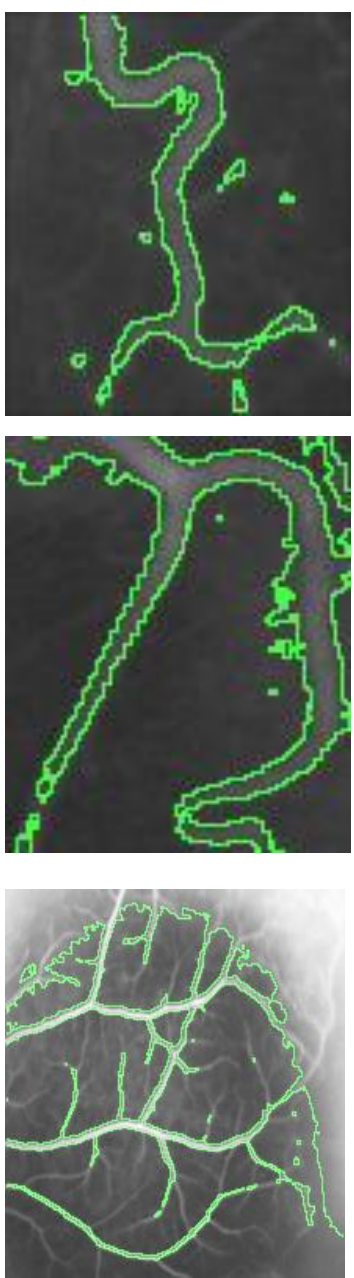

(b)
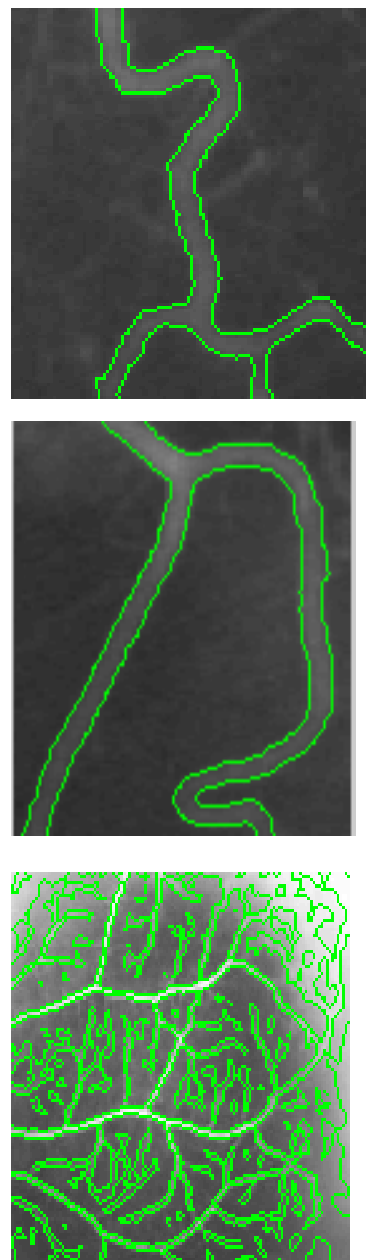

(c)

Fig. 5. Segmentation results: (a). original images, (b). model of Chan\&Vese, (c). our model.

\section{Conclusion}

In this paper we have presented a method of active contours based approach regions for the segmentation of images within the framework of the formulation level sets. The proposed method has shown its effectiveness for the segmentation of images with various intensities. Thus, the practical results show the desired performances of our model in the case of the images with contours of low contrast. The comparison with the model of Chan \& Vese shows the interests of our model such as robustness, speed and precision.

\section{References}

1. V. Caselles, R. Kimmel and G. Sapiro, « Geodesic Active Contours », International Journal of Computer VisionIJCV, vol.22, n 1 , pp.61-79, 1997.

2. L. Cohen and I. Cohen, «Finite-element methods for active contour models and balloons for 2-D and 3-D images ", IEE Trans. Patt. Anal. Mach. Intell, pp.11311147,1993

3. M. Kass, A. Witkin, D. Terzopoulos, « Snakes: Active Contours Models », Proceedings of the First International Conference on Computer Vision, pp.259-268, 1987.

4. C. Li, J. Liu, and M. D. Fox, « Segmentation of external force field for automatic initialization and splitting of snakes», Pattern Recognition, vol.38 (11), pp.1947-1960, 2005.

5. C. Li, C. Xu, C. Gui, and M. D. Fox, « Level set evolution without re-initialization: A new variational formulation », In IEEE Conference on Computer Vision and Pattern Recognotion (CVPR), vol.1, pp.430-436, 2005.

6. R. Malladi, J.A. Sethian, and B.C. Vemuri, "Shape modelling with front propagation: A level Set 
Approach », IEEE Transaction on Pattern Analysis and Machine Intelligence, vol.17, $\mathrm{n}^{\circ} 2$, pp.158-175, 1995.

7. C. $\mathrm{Xu}$ and J.L. Prince, "Snakes, Shapes, and Gradient Vector Flow », IEE Trans. on Image Processing, 1997.

8. T.F. Chan and L.A. Vese, " Active Contours Without Edges », IEEE Transactions on Image Processing, vol.10, n ${ }^{\circ}$, pp.266-277, 2001.

9. N. Paragios and R. Deriche, « Geodesic active regions and level set methods for supervised texture segmentation ", Int'lJ. Comp. Vis., vol.46, pp.223-247, 2002.

10. A. Tsai, A. Yezzi, and A. S. Willsky, « Curve evolution implementation of the Mumford-shah functional for image segmentation, denoising, interpolation, and magnification», IEEE Trans Im Proc, vol.10, pp.11691186,2001

11. A. Azizi, K. Elkourd, «Fast Region-based Active Contour Model Driven by Local Signed Pressure Force », ELCVIA Electronic Letters on Computer Vision and Image Analysis, 15(1), 1-13, 2016.

12. A. Dervieux, F. Thomasset, «A finite element method for the simulation of Rayleigh-Taylor instability ", Lecture Notes in Mathematics, vol. 771 pp. 145-159, 1979.

13. A. Dervieux, F. Thomasset. «Multifluid incompressible flows by a finite element method ", Lectures Notes in Physics, vol. 141, pp. 158-163, 1981.

14. S. Osher and J.A. Sethian, «Front propagating with curvature-dependent speed: Algorithms based on Hamilton-Jacobi formulation », Journal of Computational Physics, Vol.79, pp. 12-49, 1988.

15. J.A. Sethian, « Level Set Methods and Fast Marching Methods ", Department of Mathematics, University of California, Berkeley, Cambridge University Press, 1999.

16. D. Mumford and J. Shah, «Optimal approximations by piecewise smooth functions and associated variational problems », Commun. Pure Appl.Math., vol. 42, pp.577685, 1989.

17. C. Li, C.Y. Kao, J.C. Gore, and Z. Ding, « Minimization of Region-Scalable Fitting Energy for Image Segmentation », IEEE Transactions On Image Processing, vol. 17 , no. $10,2008$.

18. A. Abdallah, « Detection of Active Contour of Different Images ", Thesis submitted to obtain the degree of Doctorate 3rd cycle LMD in: Electrical Engineering, Option: Signals and Communications, University of Biskra, October 2017.

19. C. Li, R. Huang, Z. Ding, J.C. Gatenby, D.N. Metaxas, and J.C. Gore, « A Level Set Method for Image Segmentation in the Presence of Intensity Inhomogeneities with Application to MRI »,IEEE Transactions On Image Processing, vol. 20, no. 7, 2011.

20. L. Sun , X. Meng, J. Xu , and Y. Tian, «An Image Segmentation Method Using an Active Contour Model Based on Improved SPF and LIF», journal of Applied Sciences, December 2018.

21. L. Vese and T. Chan, «A multiphase level set framework for image segmentation using the Mumford and shah model, Int'1 J. Comp. Vis., vol.50, pp.271-293, 2002.

22. C. Li , C.Y. Kao , J .C. Gore, and Z. Ding, «Implicit Active Contours Driven by Local Binary Fitting Energy», 2007 IEEE Conference on Computer Vision and Pattern Recognition, page(s):1 - 7, 17-22 June 2007.

23. B. Chen, Q.H. Zou, W.S. Chen, and Y. Li, "A Fast Region-Based Segmentation Model with Gaussian Kernel of Fractional Order », Hindawi Publishing Corporation Advances in Mathematical Physics, 2013.

24. A. Azizi, K. Elkourd, Z. Azizi, «Robust Active Contour Model Guided by Local Binary Pattern Stopping Function », Cybernetics and Information Technologies, Volume 17, No 4, Sofia, 2017.

25. R. Dib, K. Mokrani, and A. Mekhmoukh, « Modèle de Chan et Vese avec terme de lissage : application en imagerie médicale», International Conference on Electronics \& Oil (ICEO'11), Ouargla, Algeria, 01-02 Mars 2011.

\section{Creative Commons Attribution License 4.0 (Attribution 4.0 International, CC BY 4.0)}

This article is published under the terms of the Creative Commons Attribution License 4.0 https://creativecommons.org/licenses/by/4.0/deed.en US 